\title{
Robust face recognition technique using Gabor phase pattern and phase only correlation
}

\author{
J. S. Arjun • Madhu S. Nair
}

Received: 24 February 2014/ Accepted: 4 July 2014/Published online: 1 August 2014

(C) CSI Publications 2014

\begin{abstract}
A robust and efficient method for face recognition using phase only correlation (POC) is proposed in this paper. To achieve efficient recognition rate, it uses the concept of histogram of Gabor phase pattern (HGPP) supplemented by POC technique. In HGPP, the quadrantbit codes are first extracted from faces, and in order to encode the phase variations, global Gabor phase pattern (GGPP) and local Gabor phase pattern (LGPP) are derived. GGPP and LGPP are then split into the non-overlapping rectangular regions. From the above regions, spatial histograms are extracted and concatenated into an extended histogram feature to represent the original image. The recognition is carried out with the nearest-neighbor classifier, using the histogram intersection as the similarity measurement. Finally, face patterns are verified with POC based matching technique to improve the accuracy of the system. This method improves the result both distribution wise and content wise. Experiments are done on the large scale ORL, YALE, FERET and DCSKU databases. Experimental results show that the proposed method is promising and is comparable with the advanced face recognition algorithms reported in the literature.
\end{abstract}

Keywords Feature extraction - Gabor - Gabor phase pattern $\cdot$ Phase only correlation

\footnotetext{
J. S. Arjun · M. S. Nair ( $\square)$

Department of Computer Science, University of Kerala, Kariavattom, Thiruvananthapuram 695581, Kerala, India e-mail: madhu_s_nair2001@yahoo.com

J. S. Arjun

e-mail: arjun4206@gmail.com
}

\section{Introduction}

Identifying a person poses a serious challenging task for computers. There are various recognition system developed in different areas such as fingerprint recognition, face recognition, speech recognition and iris recognition. Due to the different commercial and law enforcement applications, the face recognition has become one of the most interesting research topics. Face recognition is challenging since it is a real world problem. Human face does not have easily identifiable features and edges and consequently its recognition becomes a complex task. It is difficult to develop an accurate mathematical model for face recognition that can be kept as a reference for the study of the image. Aging, shape, pose, poor lighting, occlusion, sunglasses, long hair, occlusion and low resolution are some of the major factors that influence the face recognition problem [1].

Researchers have included various dimensionality reduction technique such as principal component analysis (PCA) [2], linear discriminant analysis (LDA) [3], independent component analysis (ICA) [1], discriminant common vector (DCV) [4], kernel-PCA, kernel-LDA [5], kernel-DCV [6], etc. for the enhancements of accuracy of face recognition. In order to train the classifiers for the face recognition, techniques such as SVM, Adaboost, Neural Network and Bayesian Network are used. In these methods, multiple training images for each subject is required in addition to the corresponding dimensionality reduction techniques to obtain an accurate output. In real time situations, multiple images for each subject may not be available.

Several variations are encoded as intensity such as albedo and shape of the object, lighting, etc. Different feature extraction and selection methods are used for the 
purpose of eliminating the external components. Marr and Hildreth [7] first introduced the Laplacian of Gaussian (LoG) in order to achieve the goal of deriving the features at a particular stage. This method was introduced to simulate the lateral inhibition for edge detection. For the increase in the efficiency of the descriptor, the feature extractor must be anisotropic. This property simultaneously enhances the feature and orientation to a certain desired value. The Gabor transformation has been mostly used in the field of image processing and the pattern recognition tasks recently [8, 9]. In Gabor wavelet [10], Gaussian envelope modulates the sinusoidal plane wave with a certain frequency and orientation. The spatial frequency structure as well as the information of the spatial relations in the image can be characterized with the wavelet. It is also adaptable for the extraction of the orientation dependent frequency contents of the patterns.

The Gabor wavelet for the face recognition using the dynamic link architecture (DLA) framework is proposed by Lades et al. [11]. Wiskott et al. [9] proposed a Gabor wavelet based elastic bunch graph matching (EBGM) method to recognize and label the human faces. In this method, the face is represented as a graph where each node consists of a cluster of coefficients called as a jet. He measured various dimensions of the face using a distance vector based on the edge of the graph. Liu and Wechsler [8] discussed a high accuracy face recognition system based on the Gabor phase features. Shan et al. [12] proposed an AdaBoost based strategy to select the discriminative features from the magnitude part of the Gabor feature, and trained with a Fisher classifier to make a final classification. Gabor wavelet has many applications in the areas such as data compression [14], optical character recognition (OCR) [15], texture analysis [16], fingerprint recognition [15], and so on. The Gabor phase feature has been used efficiently in iris and palm print identification $[17,18]$.

The histogram method has been widely adopted for analyzing and recognizing facial images [19, 20]. This method is robust to noise and local image transformations. Swain and Ballard [21] proposed the first work where the histogram is used for identification of 3D objects. Even though traditional recognition systems use histogram method in many applications, this method has some limitations since it suffers losing the structural information of the object. Multiresolution histograms have been proposed to encode the structure information by convolving the image with Gaussian filters, difference of Gaussian or Gabor filters [19].

The novel approach based on local binary pattern (LBP) is proposed by Ahonen et al. [20]. This method utilized the features such as shape and texture related to the representation of face images. The use of LBP features is based on the idea that the face images may be treated as a composition of micro-patterns. The magnitude of the Gabor feature and the LBP operator was combined by Zhang et al. [19], in which the local Gabor binary pattern histogram sequence (LGBPHS) is discussed. This method gave better performance compared with other face recognition systems using the standard databases. Both the methods were based on the spatial histogram. This can efficiently capture the structural information of the face image and also it provides a good matching strategy. The limitation of LGBPHS method is that it gives low recognition rate when applied on faces with different anomalies such as poor lighting conditions, expressions, accessories and different complex backgrounds.

In this paper, we propose a robust face recognition method based on the efficient object descriptor of histogram of Gabor Phase Pattern (HGPP) [22, 23], supplemented by phase only correlation (POC). HGPP generates a Gabor phase pattern (GPP) based on the combination of the spatial histograms and the Gabor phase information encoding scheme. The direct extraction of the features without the use of the training procedure makes this method different from other learning-based face recognition methods. GPP captures the variations that are extracted from the change in orientation of the Gabor wavelet at particular frequency. GPP is further classified into GGPP and LGPP, where GGPP captures the variations that are derived from the change in orientation of Gabor wavelet at a particular frequency and LGPP encodes the local neighborhood variations by using a novel local XOR pattern (LXP) operator. Both GGPP and LGPP are then split into the rectangular non-overlapping regions. Now, the spatial histograms are extracted and concatenated into an extended histogram feature to represent the original image. Finally, the recognition is carried out with the nearest-neighbor classifier, using the histogram intersection as the similarity measurement. After finding out the similarity measurement, we will get a candidate image matched with the database image. The matched image is further verified using the POC technique to improve the recognition rate and rejection rate of the proposed system.

The rest of this paper is organized as follows. Sect. 2 discuss about the face representation using GPP. Sect. 3 introduces the proposed method and Sect. 4 presents the details experimental analysis of the proposed method after applying it on different standard datasets. Conclusions are drawn in Sect. 5.

\section{Face representation using GPP}

In this section, we present the local feature based image representation method using Gabor phase differences 
between the referencing pixel and its neighboring pixels at each scale and orientation, with phase difference definition and encoding rules. Gabor wavelets are biologically motivated convolution kernels in the shape of plane waves, restricted by a Gaussian envelope function [9]. The standard form of a $2 \mathrm{D}$ Gabor filter is defined as:

$\psi_{u, v}(z)=\frac{\left\|k_{u, v}\right\|^{2}}{\sigma^{2}} e^{\frac{-\left\|k_{u, v}\right\|^{2}\|z\|^{2}}{2 \sigma^{2}}}\left[e^{i k_{u, v}^{z}}-e^{\frac{-\sigma^{2}}{2}}\right]$

where $\quad \overrightarrow{k_{u, v}}=\left(\begin{array}{c}k_{j} x \\ k_{j} y\end{array}\right)=\left(\begin{array}{c}k_{v} \cos \phi_{u} \\ k_{v} \sin \phi_{u}\end{array}\right), k_{v}=\frac{f_{\max }}{2^{v / 2}}, \phi_{u}=$ $u(\pi / 8), \quad v=\left[0, \ldots, v_{\max -1}\right], u=\left[0, \ldots, u_{\max -1}\right], v_{\max }=$ $5, u_{\max }=8$ and $\sigma=2 \pi . v$ is the frequency, $u$ is the orientation and $\sigma$ determines the ratio of the Gaussian window width to wavelength [11]. Gabor filter is a product of a Gaussian envelope and a complex plane wave. The first term in the square brackets in (1) determines the oscillatory part of the Gabor filter and the second term compensates for the DC value. The Gabor transformation of a given face image is defined as its convolution with the Gabor filter

$G_{u, v}(z)=I(z) * \psi_{u, v}(z)$

where $I$ is the input image, $\mathrm{z}=(\mathrm{x}, \mathrm{y})$ is the face image position and $*$ indicates the convolution operator. $G_{u, v}(z)$ is the convolution result corresponding to the Gabor filter at frequency $v$ and orientation $u$. The Gabor filter coeficient $G_{u, v}(z)$ is a complex, which can be rewritten as [24] :

$G_{u, v}(z)=A_{u, v}(z) \cdot e^{i \theta_{u, v}(z)}$

with one magnitude term $A_{u, v}(z)$, and one phase term $\theta_{u, v}(z)$. The magnitude varies slowly with spatial position, while the phase rotate in a particular rate with positions. Visualization of the Gabor magnitude and phase [22] are shown in Fig. 1. Table 1 discuss about various parameters of Gabor wavelets. Sets of Gabor wavelets for different orientations are discussed in Table 2.

\section{Proposed face recognition technique}

In this section, we propose a robust face recognition method based on effective object descriptor HGPP with POC. HGPP method is based on the combination of the spatial histogram and Gabor phase information encoding scheme. To encode the Gabor phase information two methods are used; GGPP and LGPP. GGPP and LGPP are defined based on the quadrant-bit codes of Gabor real and imaginary parts, $P_{u, v}^{R e}(Z), P_{u, v}^{I m}(Z)$, proposed by Daugman for iris recognition [18]. GGPP encodes the orientation information at each frequency and LGPP encodes the local neighborhood variations at each orientation and frequency.

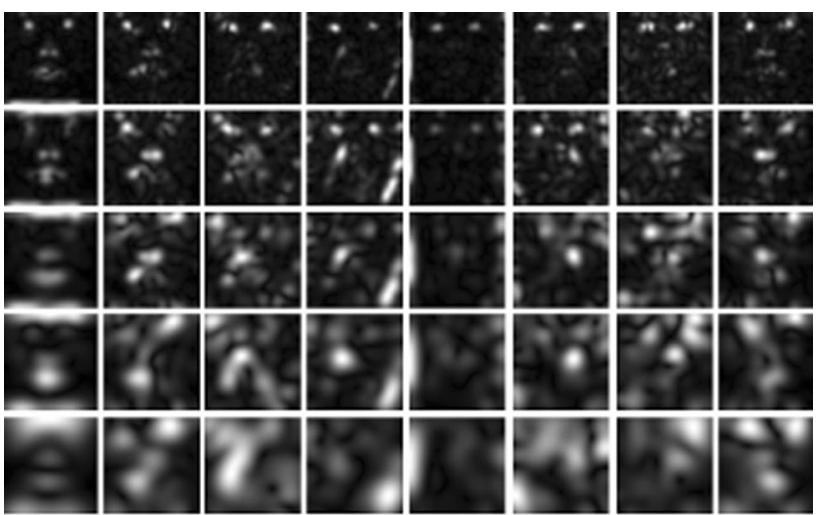

(a)

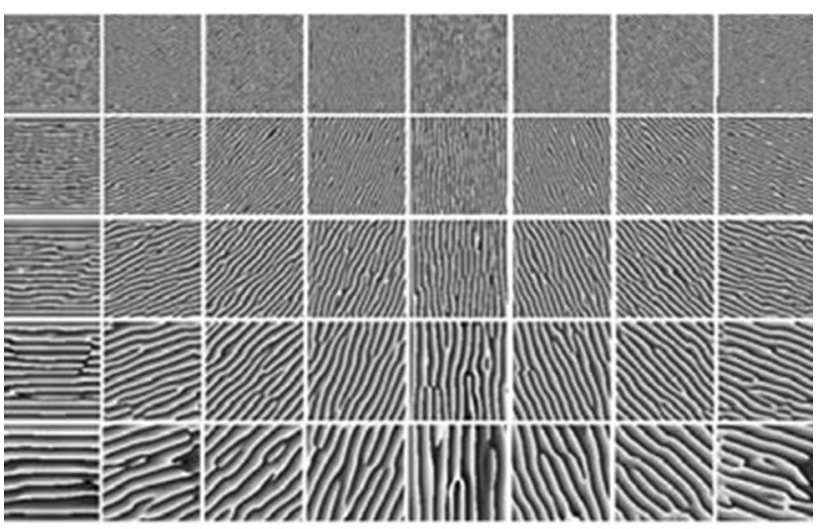

(b)

Fig. 1 Visualization of the Gabor magnitude and phase. a Gabor magnitude. b Gabor phase

Table 1 Units for magnetic properties

\begin{tabular}{lll}
\hline Parameter & Symbol & Values \\
\hline Orientation & $\theta$ & $\left\{0, \frac{\pi}{8}, \frac{2 \pi}{8}, \frac{3 \pi}{8}, \frac{4 \pi}{8}, \frac{5 \pi}{8}, \frac{6 \pi}{8}, \frac{7 \pi}{8}\right\}$ \\
Wavelength & $\lambda$ & $\{4,4 \sqrt{2}, 8,8 \sqrt{2}, 16\}$ \\
Phase & $\varphi$ & $\left\{0, \frac{\pi}{2}\right\}$ \\
Gaussian radius & $\sigma$ & $\sigma=\lambda$ \\
Aspect ratio & $\gamma$ & 1 \\
\hline
\end{tabular}

Table 2 Sets of Gabor wavelets for different orientations

\begin{tabular}{lll}
\hline No of Gabor wavelets & Values \\
\hline 5 & $\theta$ & $=0$ \\
10 & $\theta$ & $=\left\{\frac{\pi}{8}, \frac{7 \pi}{8}\right\}$ \\
15 & $\theta$ & $=\left\{0, \frac{2 \pi}{8}, \frac{4 \pi}{8}\right\}$ \\
20 & $\theta$ & $=\left\{0, \frac{2 \pi}{8}, \frac{4 \pi}{8}, \frac{6 \pi}{8}\right\}$ \\
40 & $\theta$ & $=\left\{0, \frac{\pi}{8}, \frac{2 \pi}{8}, \frac{3 \pi}{8}, \frac{4 \pi}{8}, \frac{5 \pi}{8}, \frac{6 \pi}{8}, \frac{7 \pi}{8}\right\}$ \\
\hline
\end{tabular}

They are then divided into non-overlapping rectangular regions, from which spatial histograms are extracted and concatenated into an extended histogram feature to 
represent the face image. Recognition is performed by using nearest-neighbor classifier with histogram intersection as the similarity measurement. After finding out the similarity measurement, a candidate image matching with a particular database image is obtained which is further verified using $\mathrm{POC}$ technique.

\subsection{Quadrant bit coding (QBC)}

QBC proposed in [22] is used to encode GGPP and LGPP. Quadrant-bit codes of Gabor real and imaginary parts $\left(P_{u, v}^{R e}(Z), P_{u, v}^{I m}(Z)\right)$ is based on GPP. The following rules are used for encoding the QBC [22].

$$
\begin{aligned}
P_{u, v}^{R e}(Z)=0, & \text { if } \operatorname{Re}\left(G_{u, v}(Z)\right)>0 \\
1, & \text { if } \operatorname{Re}\left(G_{u, v}(Z)\right)<=0 \\
P_{u, v}^{I m}(z)=0, & \text { if } \operatorname{Im}\left(G_{u, v}(Z)\right)>0 \\
1, \quad & \text { if } \operatorname{Im}\left(G_{u, v}(Z)\right)<=0
\end{aligned}
$$

where $\operatorname{Re}\left(G_{u, v}(Z)\right)$ and $\operatorname{Im}\left(G_{u, v}(Z)\right)$ are respectively the real and imaginary parts of the Gabor coefficient.

Daugmans encoding method, given by (4) and (5) can be reformulated as follows:

$$
\begin{aligned}
& P_{u, v}^{R e}(Z)=0 \text { if } \theta_{u, v}(z) \in\{I, I V\} \\
& 1, \quad \text { if } \theta_{u, v}(Z) \in\{I, I I I\} \\
& P_{u, v}^{I m}(Z)=0, \quad \text { if } \theta_{u, v}(Z) \in\{I, I I\} \\
& 1, \quad \text { if } \theta_{u, v}(Z) \in\{I I I, I V\}
\end{aligned}
$$

where $\theta_{u, v}(z)$ is the Gabor phase angle for the pixel at the position. It is obvious that (6) and (7), the so called QBC, assign two bits for each pixel according to the quadrant in which the Gabor phase angle lies. QBC is relatively stable, and it is actually the quantification of Gabor feature. This encoding procedure [22] can be understood more clearly as shown in Fig. 2.

\subsection{HGPP extraction and similarity measurement}

GGPP and LGPP patterns are then extracted from the QBC using the encoding and LXP techniques described in [22]. In Daugmans iris recognition method, QBC are directly used to form the representation of an iris image and the classification is achieved by hamming distance. To model them more efficiently and compactly, spatial histogram method is exploited to model the encoded GPPs (GGPP and LGPP). The reason for using histogram based approaches lies in the observations that the GPPs (both GGPP and LGPP micro-patterns) look like the images with rich structural textures and histogram serves as a good description tool for representing the texture images [25, 26]. However, a single global histogram suffers from losing

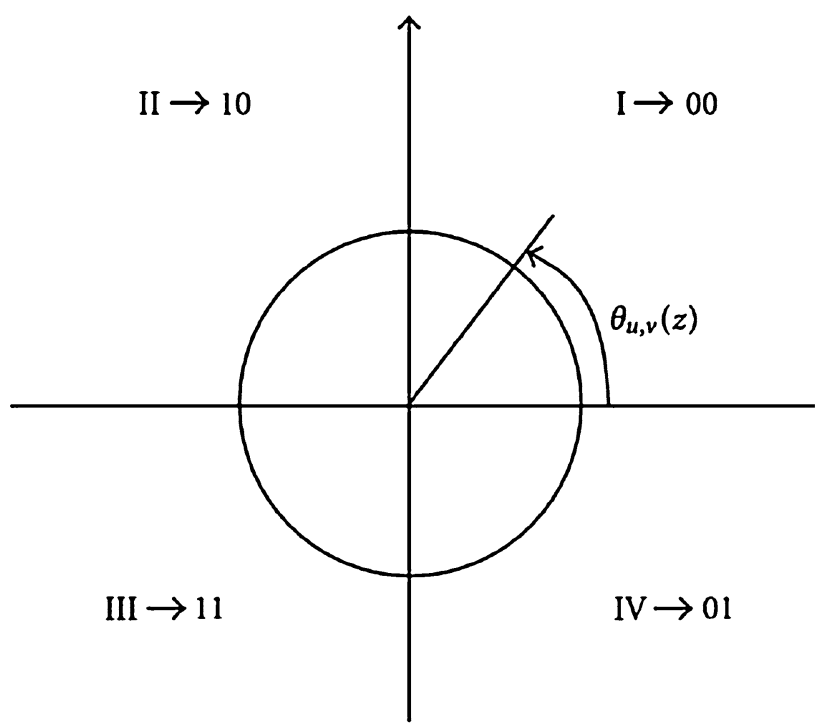

Fig. 2 Quadrant bit coding of Gabor phase

the structure information of the object. In order to preserve the spatial information in the histogram features, the GPP images are spatially divided into the non-overlapping rectangular regions, from which the spatial histograms represented are extracted. Then, all of these histograms are concatenated into a single extended histogram feature, the so-called HGPP as in [22].

As a kind of histogram-based object representation method, HGPP cannot be matched directly by the traditional distance measurements such as Euclidean distance. Although there are several methods for the histogram matching, such as histogram intersection, Chi square distance etc., in this paper, histogram intersection is being exploited as the similarity measurement [22].

Given two histograms, $\mathrm{H} 1$ and $\mathrm{H} 2$, their similarity based on histogram intersection is computed as follows:

$S(H 1, H 2)=\sum_{i=1}^{B} \min \left(H 1_{i}, H 2_{i}\right)$

where $\mathrm{B}$ is the number of bins in the histogram, $H 1_{i}$ and $H 2_{i}$ denotes the frequency of GGPP and LGPP micropatterns in the $i$ th bin, respectively.

\subsection{Phase only correlation (POC)}

When a face image is given as input to the proposed system, HGPP and histogram intersection similarity measurement finds an appropriate match with one of the images in the face database. To improve the accuracy of the proposed face recognition system, the matched image obtained from the previous phase is either accepted or rejected using a POC based decision technique. In POC based decision technique, a threshold value is used which was empirically found out 
by conducting experimental analysis on different standard databases. POC function is defined as 2D inverse Fourier transform for the cross-phase spectrum of two images [27].

Consider two images of size $M_{1} \times M_{2}$ as $f\left(m_{1}, m_{2}\right)$ and $g\left(m_{1}, m_{2}\right)$. Let $F\left(k_{1}, k_{2}\right)$ and $G\left(k_{1}, k_{2}\right)$ be their 2D discrete Fourier transforms of $f$ and $g$, respectively. Cross-phase spectrum or normalized cross spectrum $R_{F G}$ is defined as the conjugate of $G\left(k_{1}, k_{2}\right)$ multiplied by $F\left(k_{1}, k_{2}\right)$ divided by its absolute value as follows.

$R_{F G}\left(k_{1}, k_{2}\right)=\frac{F\left(k_{1}, k_{2}\right) \overline{G\left(k_{1}, k_{2}\right)}}{\left|F\left(k_{1}, k_{2}\right) \overline{G\left(k_{1}, k_{2}\right)}\right|}=e^{j \theta\left(k_{1}, k_{2}\right)}$

When 2D Inverse Fourier Transform is applied on (9), the POC function is generated as follows.

$r_{f g}\left(m_{1}, m_{2}\right)=\frac{1}{M_{1} M_{2}} \sum R_{F G}\left(k_{1}, k_{2}\right) W_{M_{1}}^{-k_{1} m_{1}} W_{M_{2}}^{-k_{2} m_{2}}$

The POC function in (10) holds some interesting properties that can be used for face recognition applications. One of the most remarkable properties of POC function, shown in Fig. 3 is that when two images are similar in nature, their POC function $r_{f g}\left(m_{1}, m_{2}\right)$ gives a distinct sharp peak. When the two images are dissimilar, the top most peak drops significantly as shown in Fig. 4. The height of the top peak is a good measure to judge the similarity between the two images. Thus the POC function exhibits much higher discrimination capability than ordinary correlation function. Figure 5 shows the flowchart of the entire working flow of the proposed system.

\section{Results and discussions}

Face recognition is one of the most challenging research topics in computer vision and patter recognition. Many

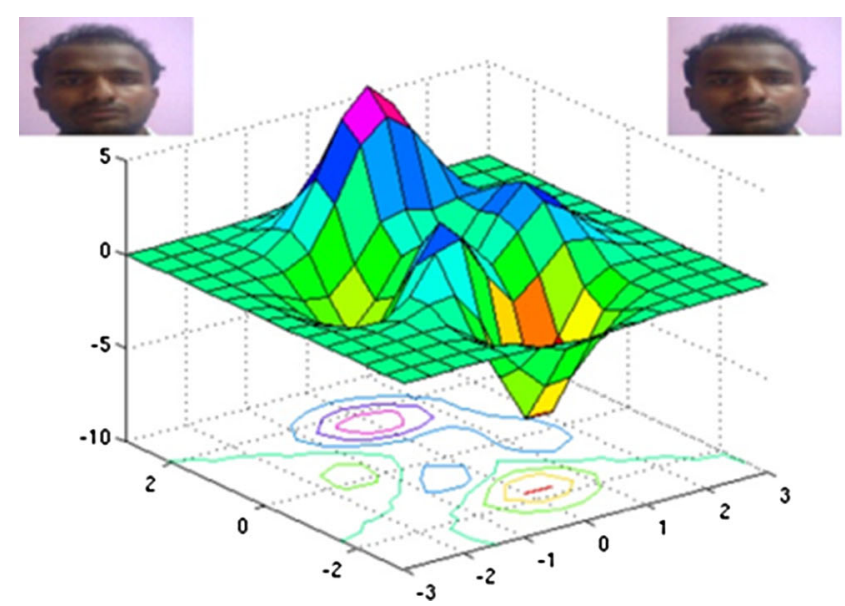

approaches, such as Eigenface [28], Fisherface [29], Gabor Fisher classifier (GFC) [11] and LGBPHS [19] are available for comparisons. In this section, we have conducted comparison experiments on 4 large-scale face databases; ORL, YALE, FERET and DCSKU database. The ORL database of faces contains ten different images of each of 40 distinct subjects. For some subjects, the images were taken at different times, varying the lighting, facial expressions and facial details. The Yale database contains 165 gray scale images in GIF format of 15 individuals. There are 11 images per subject, one per different facial expression or configuration: center-light, with glasses, happy, left-light, without glasses, normal, right-light, sad, sleepy, surprised and wink. In FERET database, Fa containing 1,196 frontal images, $\mathrm{Fb}$ containing 1195 images of expression variations and Fc containing 194 images taken under different illumination conditions, are considered. DCSKU (Department of Computer Science, Kerala University) database consists of 1,000 face images including 100 males and 50 females in different variation on lighting, expression, background and aging. In our experiments, all face images are normalized to $128 \times 128$. The system is tested using MATLAB 7.0 and is executed on Pentium- IV, 3.20 GHz processor with $1 \mathrm{~GB}$ of memory. The sample images in the ORL, YALE, FERET and DCSKU databases are shown in Fig. 6, 7, 8 and 9, respectively. Table 3 shows the comparative analysis of the proposed method with other methods based on recognition rate $\left(R_{r e c}\right)$ and rejection rate $\left(R_{r e j}\right)$. Higher recognition and rejection rate of the proposed method is due to the higher discrimination nature of POC.

\subsection{Selection of threshold value}

In the POC phase, a threshold value is set based on experimental analysis conducted on different standard

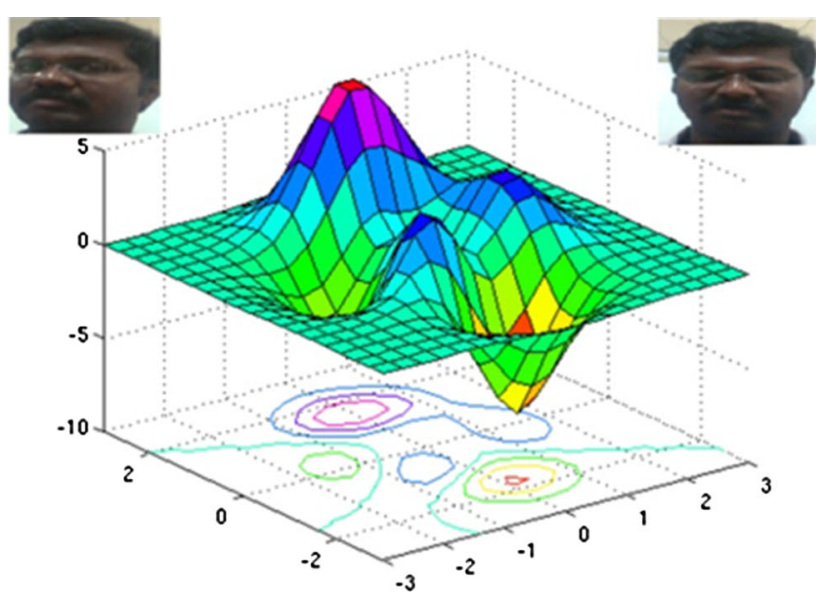

Fig. 3 Examples of POC function-distinct sharp peak for same image types 

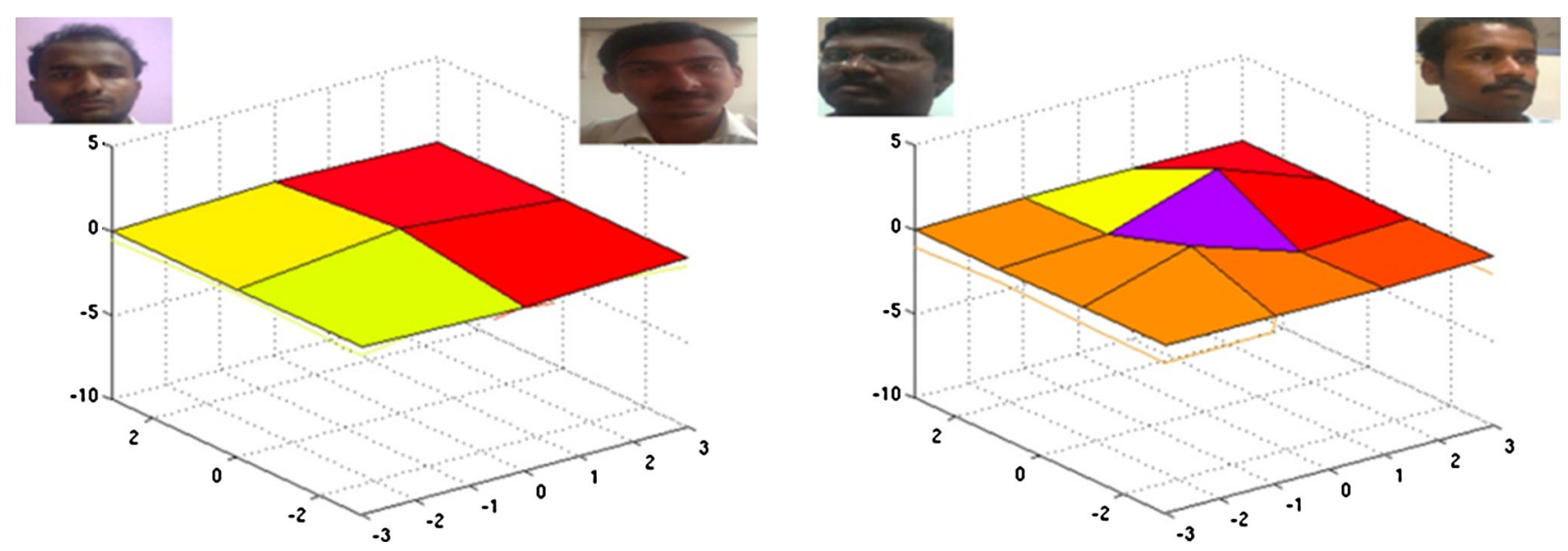

Fig. 4 Example of POC function-NO distinct peak for two different images

Fig. 5 Flowchart of the proposed method

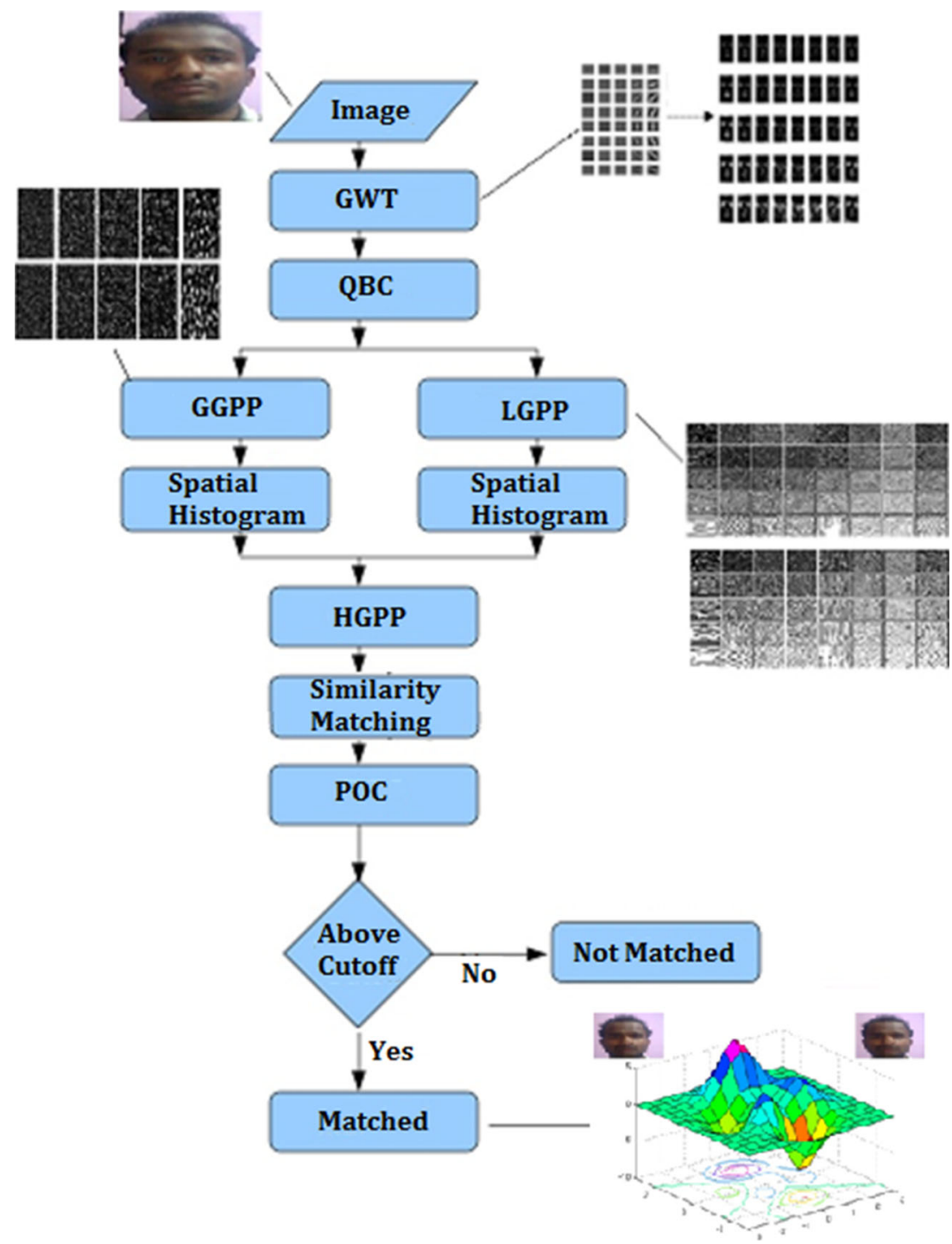



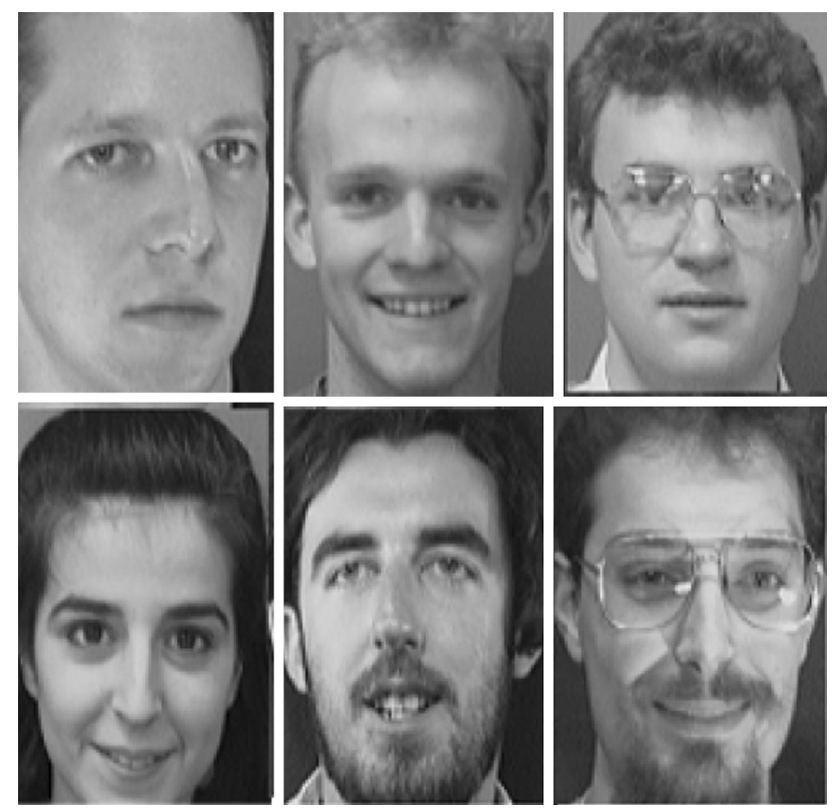

Fig. 6 Samples in the ORL database
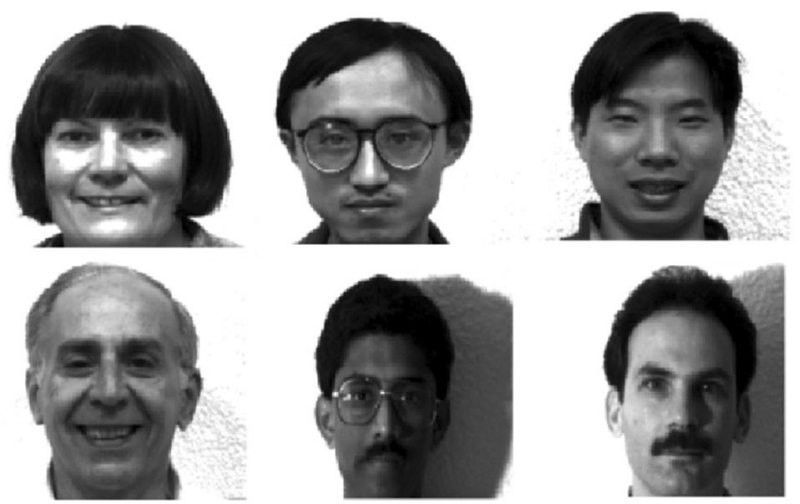

Fig. 7 Samples in the YALE database

databases. Figure 10 shows the relationship between threshold values and recognition rates. From the figure, we can conclude that higher recognition rate is achieved at a threshold value of 40 . So in all the subsequent experimental analysis, we set a threshold value of 40 .

\subsection{Experiment 1: on image size and gabor filters}

Our first experiment aims to investigate the influence of the size of the normalized face image, as well as the frequency range of the Gabor filters (parameter $f_{\max }$ ). In this experiment, we try to select a better $f_{\max }$ empirically. Three cases are considered, i.e., $128 \times 128$ image size with $f_{\max }=\pi / 2$, $88 \times 88$ image size with $f_{\max }=\sqrt{2} \pi / 2$, and $64 \times 64$ image size with $f_{\max }=\pi$. Table 4 shows the $R_{R e c}$ and $R_{R e j}$
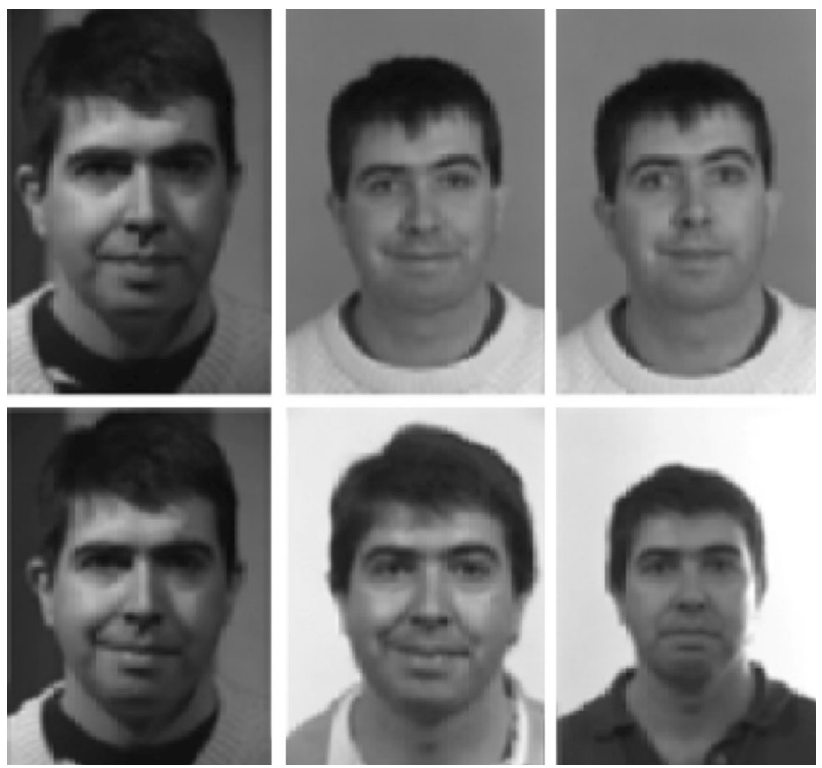

Fig. 8 Samples in the FERET database
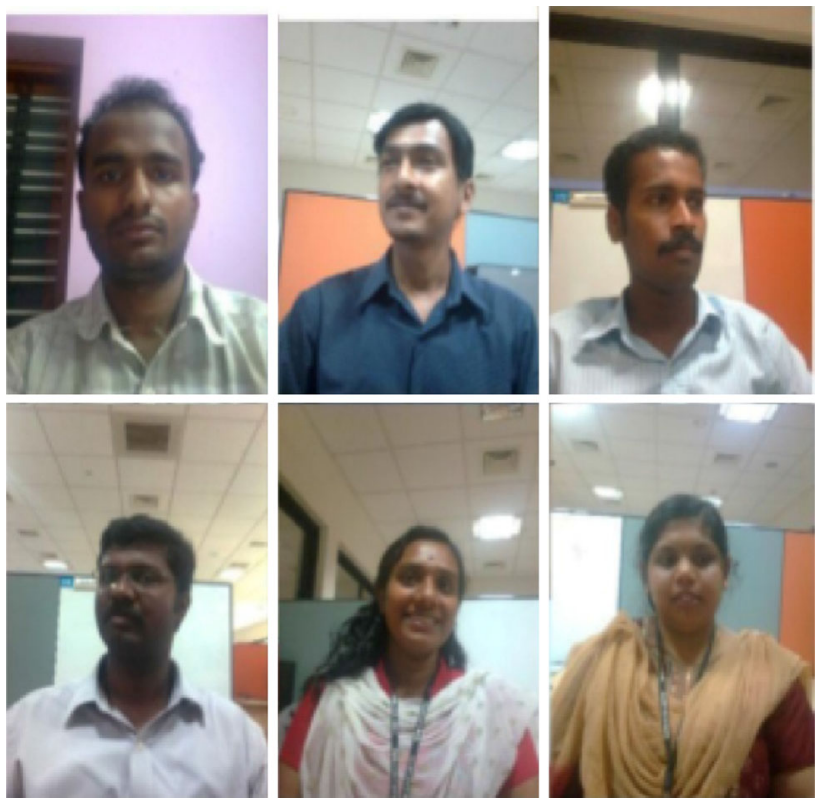

Fig. 9 Samples in the DCSKU database

of the proposed method for different sizes of the normalized images and with different values for Gabor parameter $f_{\max }$. From the table, we can conclude that HGPP using $128 \times 128$ image size with $f_{\max }=\pi / 2$ is better than that using $88 \times 88$ image size with $f_{\max }=\sqrt{2} \pi / 2$, and considerably outperforms that using $64 \times 64$ image size with $f_{\max }=\pi$. Experimental results show that the better performance can be achieved with the larger image size and an appropriate $f_{\max }$. Therefore, in the following experiments, 
Table 3 Comparison of Proposed method with other methods based on the recognition rate $\left(R_{R e c}\right)$ and rejection rate $\left(R_{R e j}\right)$

\begin{tabular}{|c|c|c|c|c|c|c|c|c|c|c|c|c|}
\hline \multirow[t]{3}{*}{ Database } & \multicolumn{12}{|c|}{ Methods } \\
\hline & \multicolumn{2}{|c|}{ Eigenface [28] } & \multicolumn{2}{|c|}{ Fisherface [29] } & \multicolumn{2}{|c|}{ GFC [11] } & \multicolumn{2}{|c|}{ LGBPHS [19] } & \multicolumn{2}{|c|}{ HGPP [22] } & \multicolumn{2}{|c|}{ Proposed method } \\
\hline & $R_{R e c}$ & $R_{R e j}$ & $R_{R e c}$ & $R_{R e j}$ & $R_{R e c}$ & $R_{R e j}$ & $R_{R e c}$ & $R_{R e j}$ & $R_{R e c}$ & $R_{R e j}$ & $R_{R e c}$ & $R_{R e j}$ \\
\hline ORL & 88.1 & 72.2 & 87.2 & 61.1 & 89.7 & 67.5 & 98.3 & - & 98.9 & - & 99.8 & 97.0 \\
\hline YALE & 85.8 & 68.7 & 81.2 & 74.4 & 86.3 & 69.9 & 96.2 & - & 97.3 & - & 98.7 & 97.1 \\
\hline FERET & 80.5 & 58.8 & 82.5 & 50.2 & 84.5 & 59.8 & 98.6 & - & 98.8 & - & 98.9 & 98.2 \\
\hline DCSKU & 50.4 & 46.3 & 54.4 & 41.6 & 58.3 & 48.2 & 93.5 & - & 93.7 & - & 96.9 & 94.2 \\
\hline
\end{tabular}

Fig. 10 Relationship between threshold value and recognition rates for the proposed method

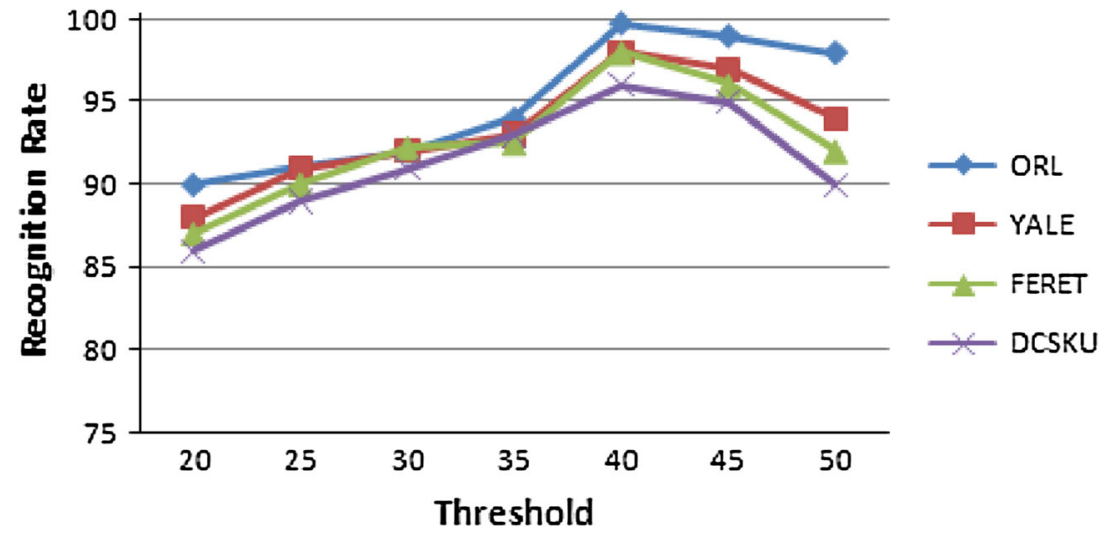

Table 4 Recognition rates $\left(R_{R e c}\right)$ and rejection rates $\left(R_{R e j}\right)$ of the proposed method for different sizes of the normalized images

\begin{tabular}{|c|c|c|c|c|c|c|}
\hline \multirow[t]{3}{*}{ Database } & \multicolumn{6}{|l|}{ Size } \\
\hline & \multicolumn{2}{|c|}{$\begin{array}{l}64 \times 64, \\
f_{\max }=\pi\end{array}$} & \multicolumn{2}{|c|}{$\begin{array}{l}88 \times 88 \\
f_{\text {max }}=\sqrt{2} \pi / 2\end{array}$} & \multicolumn{2}{|c|}{$\begin{array}{l}128 \times 128 \\
f_{\max }=\pi / 2\end{array}$} \\
\hline & $R_{R e c}$ & $R_{R e j}$ & $R_{R e c}$ & $R_{R e j}$ & $R_{R e c}$ & $R_{R e j}$ \\
\hline ORL & 98.23 & 99.32 & 99.19 & 98.42 & 99.75 & 99.43 \\
\hline YALE & 98.67 & 99.00 & 98.42 & 98.58 & 98.95 & 98.42 \\
\hline FERET & 95.27 & 95.75 & 97.26 & 94.28 & 97.34 & 94.78 \\
\hline DCSKU & 90.32 & 92.32 & 94.12 & 93.24 & 93.34 & 95.89 \\
\hline
\end{tabular}

we fix the image size to be $128 \times 128$ with the Gabor parameter $f_{\max }=\pi / 2$.

\subsection{Experiment 2: comparisons based on DCSKU evaluation protocol}

More experiments are conducted on another large-scale face database, DCSKU, for further validation of the proposed method. Currently, the DCSKU face database contains 1,000 images of 150 individuals (100 males and 50 females) with varying pose, expression, accessory, and lighting. According to the DCSKU evaluation protocol, experiments are conducted on the 4 probe sets, i.e., expression, aging, lighting and background. Table 5 discuss about experiment analysis performed on DCSKU database with different probe sets. From the table, we can conclude that the proposed method gives higher recognition rates for all the different probe sets. Figure 11 shows the samples taken from DCSKU database belonging to the different probe sets.

\subsection{Experiment 3: on the size of subregion}

After the input face image is transformed to the GPP domain, we use the sub region histograms to model the face. The advantage of the spatial histogram over the holistic histogram lies in its preservation of the spatial structure information. However, the size of the sub region has to be determined to balance the spatial locality and compactness of the model. Therefore, we do the experiments on the ORL, YALE, FERET and DCSKU databases to examine the influence of the sub region size on the recognition rate. As the previous experiments suggest, we conducted the experiments based on the images of size $126 \times 128$ with $f_{\text {max }}=\pi / 2$. Six different sub region sizes, $32 \times 64$, $32 \times 32,32 \times 16,16 \times 16,16 \times 8$ and $8 \times 8$, are tested. As expected, a too-large subregion size may degrade the system due to the loss of much spatial information as shown in Fig. 12. However, a small subregion size will also result in the increase of the model complexity. 
Table 5 Experimental analysis performed on DCSKU database with different probe sets

\begin{tabular}{|c|c|c|c|c|c|c|c|c|c|c|c|c|}
\hline \multirow[t]{3}{*}{ Probe sets } & \multicolumn{12}{|c|}{ Methods } \\
\hline & \multicolumn{2}{|c|}{ Eigenface [28] } & \multicolumn{2}{|c|}{ Fisherface [29] } & \multicolumn{2}{|c|}{ GFC [11] } & \multicolumn{2}{|c|}{ LGBPHS [19] } & \multicolumn{2}{|c|}{ HGPP [22] } & \multicolumn{2}{|c|}{ Proposed method } \\
\hline & $R_{R e c}$ & $R_{R e j}$ & $R_{R e c}$ & $R_{R e j}$ & $R_{R e c}$ & $R_{R e j}$ & $R_{R e c}$ & $R_{R e j}$ & $R_{R e c}$ & $R_{R e j}$ & $R_{R e c}$ & $R_{R e j}$ \\
\hline Accessory & 39.1 & 42.3 & 65.1 & 51.4 & 78.8 & 62.4 & 90.3 & - & 91.8 & - & 93.3 & 92.0 \\
\hline Lighting & 42.7 & 46.7 & 68.3 & 71.28 & 74.3 & 68.4 & 89.2 & - & 90.6 & - & 94.7 & 96.0 \\
\hline Expression & 58.1 & 56.7 & 71.2 & 72.2 & 80.4 & 72.8 & 88.7 & - & 92.9 & - & 98.2 & 94.2 \\
\hline Background & 80.4 & 56.3 & 65.1 & 51.6 & 71.3 & 58.9 & 85.3 & - & 93.7 & - & 98.9 & 92.2 \\
\hline
\end{tabular}
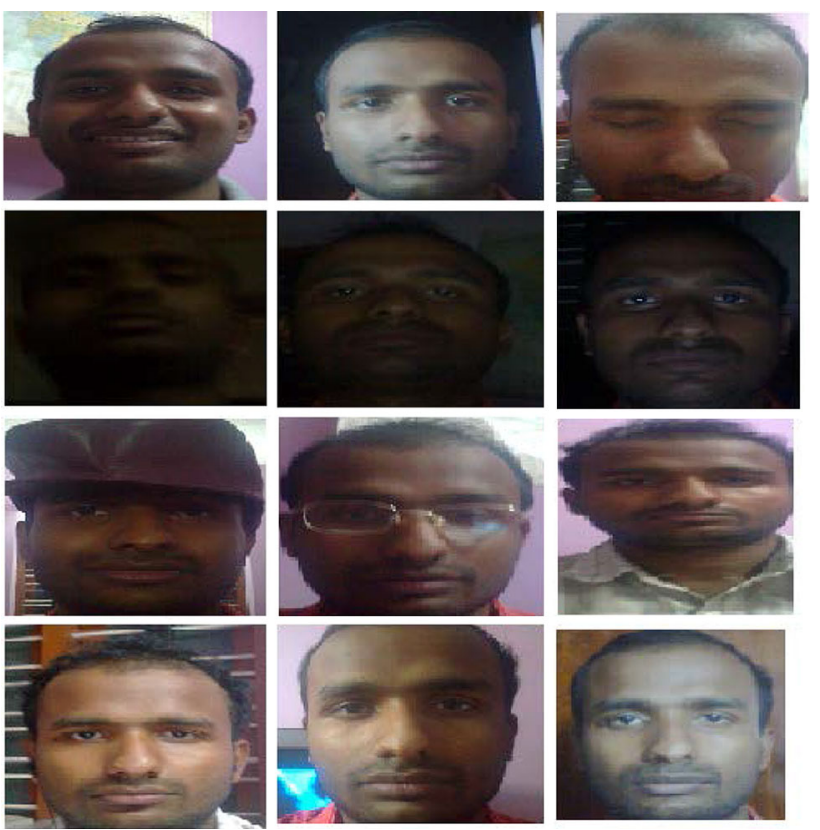

Fig. 11 Samples in DCSKU database. Rows 1, 2, 3, 4 show the expression faces, lighting faces, accessory faces and different background faces respectively

\subsection{Experiment 4: on the number of histogram bins}

Following experiments show that the performance of HGPP is also affected by the number of histogram bins. Using a small number of histogram bins makes the feature vector shorter but loses more information. The full- length histogram will also increase the model complexity and become more sensitive to the noise. One has to choose an appropriate number of bins in order to balance the efficiency and model complexity. In this paper, the uniform quantization method is used to partition the sub region histogram with equal intervals, i.e., $[0, \ldots, 256 / \mathrm{B}-1]$, $[256 / \mathrm{B}, \ldots, 2 \times 256 / \mathrm{B}-1], \ldots .,[256-256 / \mathrm{B}, \ldots ., 255]$, where $\mathrm{B}$ represent the number of histogram bins. It is obvious that the length of the histogram feature will be greatly reduced when the number of histogram bins is changed from 256 to 16 . However, the performance does not suffer a great deal. For example, changing from 256 bins to 32 bins, the recognition rate drops only from 99.75 to $97.5 \%$ on the ORL database. Figure 13 shows the relationship between the number of histogram bins and recognition rates.

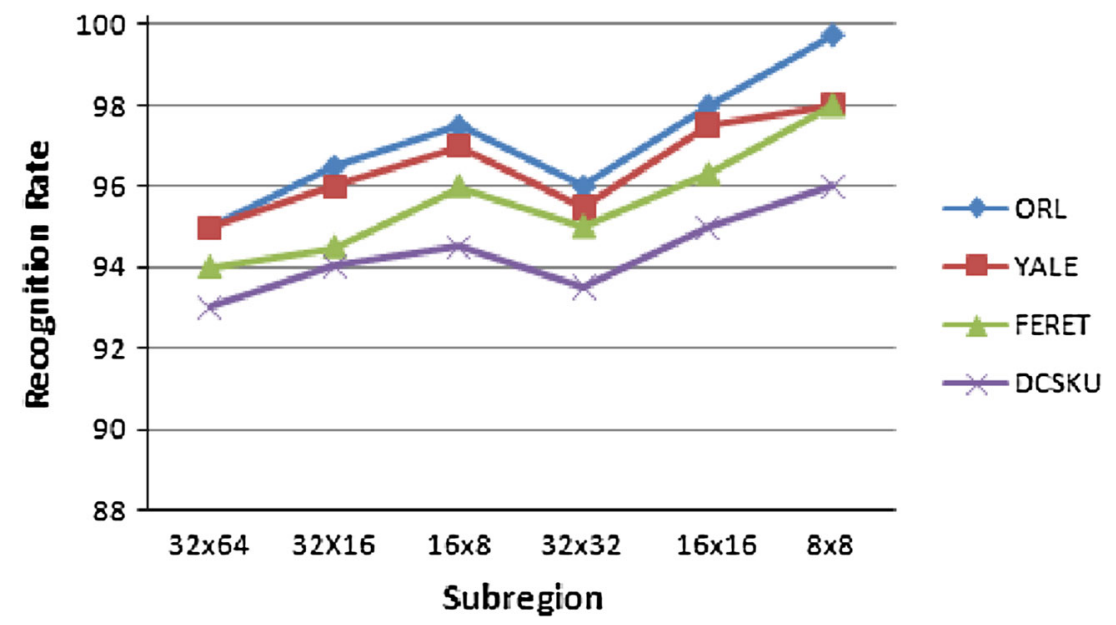

Fig. 12 Recognition rates of the proposed method for different sub regions values 
Fig. 13 Relationship between the numbers of histogram bins and recognition rates for the proposed method

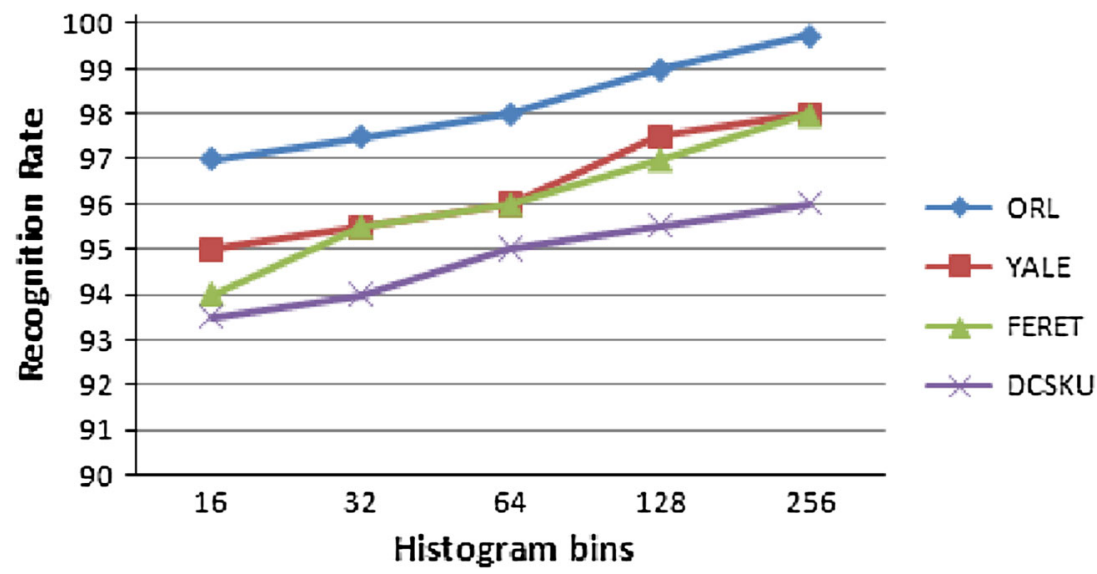

Table 6 Comparison of proposed method with other state-of-the-art methods based on recognition rate $\left(R_{R e c}\right)$ and rejection rate $\left(R_{R e j}\right)$

\begin{tabular}{|c|c|c|c|c|c|c|c|c|c|c|}
\hline \multirow[t]{3}{*}{ Database } & \multicolumn{10}{|c|}{ Methods } \\
\hline & \multicolumn{2}{|c|}{ Linear regression } & \multicolumn{2}{|l|}{ LDP } & \multicolumn{2}{|c|}{ Age-invariant } & \multicolumn{2}{|l|}{ LTF } & \multicolumn{2}{|c|}{ Proposed method } \\
\hline & $R_{R e c}$ & $R_{R e j}$ & $R_{R e c}$ & $R_{R e j}$ & $R_{R e c}$ & $R_{R e j}$ & $R_{R e c}$ & $R_{R e j}$ & $R_{R e c}$ & $R_{R e j}$ \\
\hline ORL & 93.14 & 91.32 & 92.29 & 90.12 & 91.71 & 92.18 & 97.13 & 94.22 & 99.75 & 97.00 \\
\hline YALE & 92.65 & 88.72 & 94.12 & 89.18 & 85.18 & 80.17 & 95.23 & 93.12 & 98.71 & 97.12 \\
\hline FERET & 88.41 & 85.66 & 88.16 & 85.22 & 88.33 & 89.12 & 96.67 & 90.15 & 98.93 & 98.16 \\
\hline DCSKU & 77.22 & 84.12 & 75.24 & 70.67 & 80.14 & 75.28 & 94.62 & 90.34 & 96.91 & 94.23 \\
\hline
\end{tabular}

4.6 Experiment 5: comparative analysis of the proposed method with other state-of-the-art methods

The performance of the proposed method has been compared with other advanced methods already reported in the literature. Some of the state-of-the-art methods reported in the literature are Linear Regression for Face Recognition [30], local derivative pattern (LDP) versus LBP [31], Ageinvariant face recognition [32] and local texture feature (LTF) [33].

From the comparative analysis, it has been found that our proposed method gives better recognition and rejection rate compared to other advanced methods. Performance analysis of advanced methods as well as the proposed method (in terms of recognition and rejection rate) is shown in the Table 6. From the table it can be concluded that our proposed method outperforms advanced methods reported in the literature.

\section{Conclusion}

A novel face recognition system is proposed here based on Gabor phase feature and POC. A good recognition and rejection rate has been achieved by the proposed method, especially in the case of face images with different anomalies. The major features of the proposed method are as follows; (i) Since HGPP is not a learning-based face recognition method, the problems faced by the traditional learning-based methods is avoided, (ii) rather than using Gabor magnitude, Gabor phase has been used in HGPP in order to derive intrinsic object information, and (iii) POC improves the recognition and rejection rate of the proposed system. Experiments are conducted using large scale databases such as ORL, YALE, FERET and DCSKU. From the experimental analysis, it can be concluded that proposed method yields better recognition rate and accuracy than other methods.

\section{References}

1. Kotani K, Qiu C, Ohmi T (2002) Face recognition using vector quantization histogram method. Proc Int Conf Image Process 2:105-108

2. Turk MA, Pentland AP (1991) Face recognition using Eigenfaces.In: Proceedings of computer vision and pattern recognition, pp 586-591

3. Martinez M, Kak AC (2001) PCA versus LDA. IEEE Trans Pattern Anal Mach Intell 23(2):228-233

4. Moeslund TB, Granum E (2001) A survey of computer visionbased human motion capture. Comput Vis Image Understand 81(3):231-268 
5. Yang MH (2002) "Kernel Eigenfaces vs. Kernel Fisherfaces: face recognition using kernel methods. In: Proceedings of international conference on automatic face and gesture recognition, May 2002, pp 215-220

6. He YH, Zhao L, Zou CR (2005) Kernel discriminative common vectors for face recognition. In: Proceedings of international conference on machine learning and cybernetics, pp 4605-4610

7. Marr D, Hildreth E (1980) Theory of edge detection. Proc R Soc Lond B 207:187-217

8. Liu C, Wechsler H (2002) Gabor feature based classification using the enhanced Fisher linear discriminant model for face recognition. IEEE Trans Image Process 11(4):467-476

9. Wiskott L, Fellous J-M, Kuiger N, von der Malsburg C (1997) Face recognition by elastic bunch graph matching. IEEE Trans Pattern Anal Mach Intell 19(7):775-779

10. Lee TS (1996) Image representation using 2D Gabor wavelets. IEEE Trans Pattern Anal Mach Intell 18(10):959-971

11. Lades M, Vorbruggen JC, Buhmann J, Lange J, von der Malsburg C, Wurtz RP, Konen W (1993) Distortion invariant object recognition in the dynamic link architecture. IEEE Trans Comput 42(3):300-311

12. Shan S, Yang P, Chen X, Gao W (2005) AdaBoost Gabor Fisher classifier for face recognition. In: Proceedings of IEEE international workshop analysis and modeling of faces and gestures, pp 278-291

13. Szu H, Telfer B, Garcia J (1996) Wavelet transforms and neural networks for compression and recognition. Neural Netw 9(4):695-708

14. Chen X, Yang J, Zhang J, Waibel A (2004) Automatic detection and recognition of signs from natural scenes. IEEE Trans Image Process 13(1):87-99

15. Mehrotra R, Namuduri K, Ranganathan N (1992) Gabor filterbased edge detection. Pattern Recognit 25(12):1479-1494

16. Lee J, Wang SD (1999) Fingerprint feature extraction using Gabor filters. Electron Lett 35(4):288-290

17. Daugman JG (1993) High confidence visual recognition of persons by a test of statistical independence. IEEE Trans Pattern Anal Mach Intell 15(11):1148-1161

18. Zhang D, Kong WK, You J, Wong M (2003) Online palm print identification. IEEE Trans Pattern Anal Mach Intell 25(9): $1041-1050$

19. Zhang W, Shan S, Gao W, Chen X, Zhang H (2005) Local Gabor binary pattern histogram sequence (LGBPHS): a novel non- statistical model for face representation and recognition. In: Proceedings of the 10th IEEE international conference on computer vision, pp 786-791

20. Ahonen T, Hadid A, Pietikainen M (2004) Face recognition with local binary pattern. In: Proceedings of the 8th European conference on computer vision, pp 469-481

21. Swain MJ, Ballard DH (1991) Color indexing. Int J Comput Vis 7(1):11-32

22. Zhang B, Shan S, Chen X, Gao W (2007) Histogram of Gabor phase patterns (HGPP): a novel object representation approach for face recognition. IEEE Trans Image Process 16(7):57-68

23. Xie S, Shan S, Chen X, Chen J (2010) Fusing local patterns of Gabor magnitude and phase for face recognition image processing. IEEE Trans Image Process 19(5):1349-1361

24. Gabor D (1946) Theory of communication. J Inst Elect Eng 93(26, pt. III):429-457

25. Zhang H, Gao W, Chen X, Zhao D (2005) Learning informative features for spatial histogram-based object detection. In: Proceedings of international joint conference neural networks, pp 1806-1811

26. Zhao W, Chellappa R, Rosenfeld A, Phillips PJ (2003) Face recognition: a literature survey. ACM Comput Surv 35(4): $399-458$

27. Ito K, Nakajima H, Kobayashi K, Aoki T, Higuchi T (2004) A fingerprint matching algorithm using phase-only correlation. IEICE Trans Fundam E87-A(3):682-691

28. Turk M, Pentland A (1991) Face recognition using eigenfaces. In: Proceedings of IEEE conference computer vision and pattern recognition, pp 586-590

29. Belhumer PN, Hespanha JP, Kriegman DJ (1997) Eigenfaces vs. fisherfaces: recognition using class specific linear projection. IEEE Trans Pattern Anal Mach Intell 19(7):711-720

30. Naseem I, Togneri R, Bennamoun M (2010) Linear regression for face recognition. IEEE Trans Pattern Anal Mach Intell 32(11):2106-2112

31. Zhang B, Gao Y, Zhao S, Liu J (2010) Local derivative pattern versus local binary pattern: face recognition with high-order local pattern descriptor. IEEE Trans Image Process 19(2):533-544

32. Park U, Tong Y, Jain AK (2010) Age-invariant face recognition. IEEE Trans Pattern Anal Mach Intell 32(5):947-954

33. Tan X, Triggs B (2010) Enhanced local texture feature sets for face recognition under difficult lighting conditions. IEEE Trans Image Process 19(6):1635-1650 